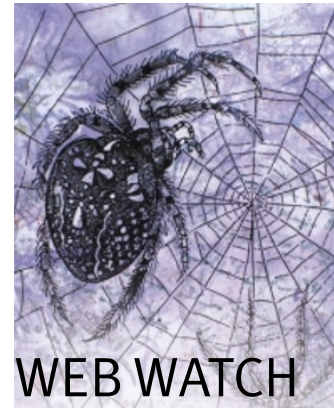

Around the worm in $\mathbf{8 0}$ ways

- WormAtlas

http://www.wormatlas.org/

- WormBase

http://www.wormbase.org/

If your research involves

Caenorhabditis elegans and you've ever dreaded the

thought of ploughing through a never-ending pile of papers to understand that unfamiliar anatomical phenotype in your model, then WormAtlas

could be the solution to your problems.

The site, which went live this year, aims to make the anatomy of $C$. elegans simple and accessible. WormAtlas is funded by a grant from the Nationa Institutes of Health and is in close collaboration with WormBase, the definitive database for genes and proteins in $C$, elegans.

WormAtlas provides an illustrated handbook of worm anatomy and a description of staining and electron-

microscopy methods, but where the site shines is the section entitled 'The slidable worm'. This site contains fine-structure images of cross-sections of the worm, a must for those research groups that do not have access to an electron microscope. This section is not yet complete, but its aim is to have $600-1,200$ annonated electronmicroscopy images in total.

The most frustrating aspect of what promises to be an invaluable resource is that many aspects are currently incomplete, including the illustrated handbook. Also, the site currently focuses on features of the wild-type adult anatomy, but it will eventually provide data on key mutant phenotypes, and the anatomy of the L1 larva and, where feasible, embryonic development. However, one future advantage of the Atlas will be that it is updateable, with submissions accepted in many formats, such as text and JPEG and TIFF images. Simon Frantz

\title{
Hedgehog in growth...
}

Mutations that activate the Hedgehog $(\mathrm{Hh})$ signalling pathway have been linked to tumour formation, but it's not been clear how. The discovery of a direct link between $\mathrm{Hh}$ signalling and key regulators of the cell cycle might now provide the answer.

Wei Du and colleagues were studying eye development in Drosophila melanogaster. The expression pattern of Hh during this process, just posterior to cells entering $S$ phase, indicated that reception of the Hh signal might be needed for entry to $S$ phase. To test this, the authors looked at what would happen if Hh signalling was blocked during eye development. They found that second mitotic wave cells with mutated smoothened (smo), a gene that is required for Hh signalling, do not enter $S$ phase. By contrast,

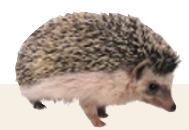

SEX DETERMINATION

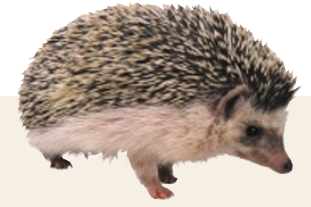

overexpression of Cubitus interruptus (Ci) - the transcription factor that mediates Hh signalling - drove G1arrested cells to enter $S$ phase.

One protein that promotes $S$ phase is Cyclin D. During eye development, the highest expression of Cyclin D overlaps with that of $\mathrm{Ci}$ - so could $\mathrm{Ci}$ promote the expression of Cyclin $\mathrm{D}$ ? Support for this idea came from the observation that levels of Cyclin D are reduced in smo-mutant clones, and also that overexpression of $\mathrm{Ci}$ induces high levels of Cyclin D messenger RNA and protein.

As well as promoting entry into $S$ phase, Cyclin D induces cell growth. $\mathrm{Du}$ and co-workers therefore wondered whether

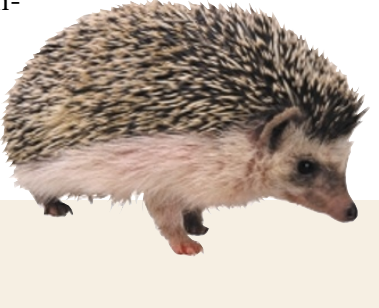

\section{... and differentiation}

A crucial event during early development of the testis is the specification of somatic cell lineages such as the Leydig cells. Little is known about the origin of fetal Leydig cells, or of the signals that induce them to differentiate. Now, however, reporting in Genes and Development, Blanche Capel and colleagues provide evidence that the Desert Hedgehog (DHH)/Patched 1 (PTCH1) pathway triggers Leydig cell differentiation.

Fetal Leydig cells are responsible for the initial masculinization of an embryo. They are first identifiable in the interstitium of $\mathrm{XY}$ gonads, where they express enzymes that are needed for the production of male sex hormones. There is some evidence that Leydig cell precursors migrate to the gonad from the mesonephros; but whatever their origin, most are
Hh might also regulate growth, so they studied the effects of overexpressing either Ci or Patched (Ptc; an inhibitor of Hh signalling) in clones of undifferentiated wing-disc cells. Whereas Ptc overexpression clones were considerably smaller than controls, Ci overexpression clones were much larger, which indicates that $\mathrm{Hh}$ signalling not only promotes $S$ phase, but that it also regulates cell growth.

Cyclin E also promotes $S$ phase, and reduced or increased levels of this protein could be detected with loss of smo or overexpression of $\mathrm{Ci}$, respectively. The authors then looked at how Hh signalling might induce the transcription of Cyclin E. They identified several sequences in the Cyclin E promoter with homology to the consensus Ci-binding site, and used chromatin immunoprecipitation to show that $\mathrm{Ci}$ indeed binds these sites in vivo. Hh signalling therefore seems to promote $S$ phase by

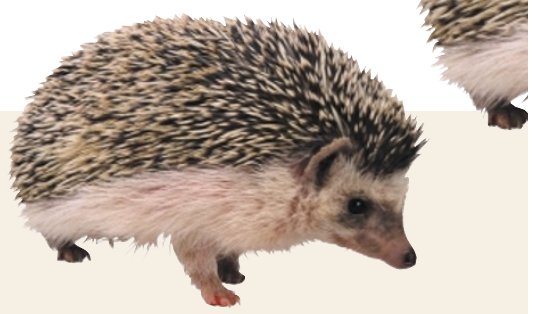

development of Leydig cells. So Capel and colleagues next asked what effect the loss of this pathway would have on Leydig cell differentiation. They analysed the expression of $S c c$ in $\mathrm{Dhh}^{+/+}, \mathrm{Dhh}^{+/}$ and $D h h^{-/} \mathrm{XY}$ gonads at $13.5-14.5$ dpc. Scc staining was seen at the centre of $\mathrm{Dhh}^{+/+}$and $\mathrm{Dhh}^{+/-}$gonads at $13.5 \mathrm{dpc}$, yet it was absent from $70 \%$ of the $\mathrm{Dhh}^{-/-}$gonads at this stage. Even by $14.5 \mathrm{dpc}$, the $D h^{-/-}$ gonads showed only very sparse staining for $S c c$.

The authors next investigated why the lack of DHH signalling leads to defects in Leydig cell differentiation. One idea was that it might affect the migration of cells from the mesonephros to the gonad. However, two independent experimental approaches showed that this migration process was normal in $D h h^{-1}$ gonads. Another possibility was that $\mathrm{DHH}$ signalling is involved in the proliferation or 


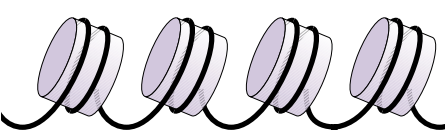

direct induction of Cyclin E expression, as well as Cyclin D.

This study shows a direct link between $\mathrm{Hh}$ signalling and cell growth (though Cyclin D) and proliferation (through both Cyclin D and Cyclin E). And, as the authors conclude, "constitutive $\mathrm{Hh}$ signalling, which promotes deregulated expression of G1-S cyclins that have been associated with diverse forms of human cancer, would promote both cell proliferation and growth in tumours".

Alison Mitchell

(2) References and links ORIGINAL RESEARCH PAPER Duman-Scheo M., Weng, L. \& Du, W. Hedgehog regulates cell growth and proliferation by inducing Cyclin D and Cyclin E. Nature 417, 299-304 (2002) WEB SITE

Wei Du's laboratory:

http://devbio.bsd.uchicago.edu/index3.html?conte t=faculty/wDu/index.htm

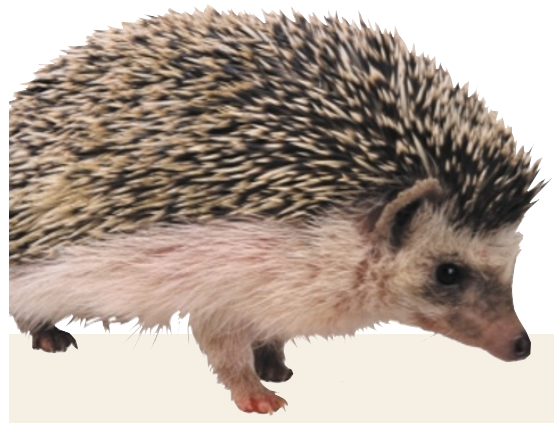

survival of fetal Leydig cell precursors in the interstitium of $\mathrm{XY}$ gonads. Again, however, there was no difference in these processes in the presence or absence of DHH signalling.

So how does the DHH pathway affect differentiation? Capel and colleagues think that its main role is in the upregulation of $S c c$ in Leydig precursor cells. Scc is the target of the steroidogenic factor 1 , and there is evidence that this, too, is upregulated in Leydig cells. By upregulating these factors, the DHH pathway could trigger the differentiation of precursors into Leydig cells. However, as the authors point out, not all cells that express Ptch 1 differentiate as Leydig cells, so other signals probably combine with the $\mathrm{DHH}$ pathway to specify Leydig cell fate.

Alison Mitchell

(2) References and links ORIGINAL RESEARCH PAPER Yao, H. H.-C. Whoriskey, W. \& Capel, B. Desert Hedgehog/Patched 1 signaling specifies Leydig cell fate in testis organogenesis. Genes Dev . 16, 1433-1440 (2002)

\section{CHROMATIN}

\section{HIRA order}

The DNA in chromatin is tightly wound around nucleosomes - but how does it get that way? Although several factors are known to deposit histones on DNA during DNA synthesis, a report in Molecular Cell now uncovers an independent chromatin-assembly pathway that is not coupled to DNA replication.

Geneviève Almouzni and colleagues were studying HIRA, a protein that was previously shown to interact with histones in mammals. They first isolated the homologue of HIRA in Xenopus laevis, and showed that GST-HIRA could bind to all four core histones $(\mathrm{H} 2 \mathrm{~A}, \mathrm{H} 2 \mathrm{~B}, \mathrm{H} 3$ and $\mathrm{H} 4)$ with varying affinities.

The authors next used a supercoiling assay to show that HIRA can facilitate the formation of nucleosomes in vitro. They mixed purified histones with a relaxed DNA plasmid in the presence of recombinant HIRA, then monitored the electrophoretic mobility of the plasmid. Nucleosome assembly causes supercoiling of the DNA, and hence faster migration on a gel.

Almouzni and co-workers then studied nucleosome assembly using Xenopus egg extracts, which are very efficient at chromatin assembly and are enriched in HIRA. Although these extracts could promote nucleosome assembly, extracts that had been immunodepleted of HIRA could not. However, depletion of HIRA did not affect the chromatinassembly activity that is coupled to nucleotide-excision repair or to DNA synthesis.

These results, conclude the authors, "show that HIRA is critical in a specific chromatin-assembly process that is not coupled to DNA synthesis". What they also emphasize is that there are at least two distinct nucleosome-assembly pathways.

(1) References and links ORIGINAL RESEARCH PAPER Ray-Gallet, D. et al. HIRA is critical for a nucleosome assembly pathway independent of DNA synthesis. Mol. Cell 1091-1100 (2002)
Alison Mitchell

\section{STRUCTURE WATCH}

\section{Open the gate...}

Ion channels open and close in response to a stimulus that 'gates' the channel. But how does gating occur, and how do pores open? In Nature, new insights have now been provided by two papers from the MacKinnon group. In the first paper, MacKinnon's group presents the structura basis of ligand gating in a $\mathrm{K}^{+}$ channel that opens in response to intracellular $\mathrm{Ca}^{2+}$. They determined the 3.3- $\AA$ resolution crystal structure of MthK from Methanobacterium thermoautotrophicum in its $\mathrm{Ca}^{2+}$-bound, open conformation. The channel is tetrameric, and the subunits that form the pore (top of figure) are each made up of two transmembrane segments. Each subunit also has a 'regulator of $\mathrm{K}^{+}$ conductance' (RCK) domain at the intracellular surface, although MthK actually has eight RCK domains (bottom of figure), as four RCK domains join the complex from the intracellular solution.

The RCK domains form a 'gating ring' through a pattern of alternate 'fixed' and 'flexible' interfaces, which actually makes four rigid units (RCK-domain dimers joined by the fixed interface). The flexible interfaces form ligand-binding clefts between RCK domains, and two $\mathrm{Ca}^{2+}$ ions (yellow circles) - which are directly correlated with channel gating - bind to each of these clefts.

By comparing the structure of the $\mathrm{Ca}^{2+}$-bound RCK domain of MthK with that of an unbound RCK domain from an Escherichia coli $\mathrm{K}^{+}$channel, the authors gained insight into how the gating ring converts the free energy of $\mathrm{Ca}^{2+}$ binding into mechanical changes in the pore. They propose that $\mathrm{Ca}^{2+}$ binding to the cleft reshapes it, so that the rigid units tilt and expand the diameter of the gating ring. This, in turn, pulls open the inner helices of the pore (see dashed lines) and lets ions pass through.

\section{...and the pore}

In the second paper, the group investigated how a pore opens by comparing the 'open' MthK structure with the known 'closed' structure of $\mathrm{KcsA}$ - a K ${ }^{+}$channel from Streptomyces lividans. Although the region around the ion selectivity filter is similar in both channels, the authors noticed large structural differences in the inner helices of the two pores.

The helices are almost straight in KcsA, and form a bundle that closes the pore near its intracellular opening. However, in MthK, the helices are bent and splayed open, which produces a wide pore. The bending point corresponds to a glycine - the most flexible amino acid - that is located deep inside the membrane, and MacKinnon's group found that this 'hinge' residue is conserved in a wide range of $\mathrm{K}^{+}$channels.

These observations fit neatly with the gating mechanism described above - ligand binding reorganizes the gating ring, which exerts a radial force that focuses at the hinge and causes the inner helices of the pore to bend outwards, thus opening the pore. REFERENCES Jiang, Y. et al. Crystal structure and mechanism of a calcium-gated potassium channel. Nature 417, 515-522 (2002) | Jiang, Y. et al. The open pore conformation of potassium channels. Nature 417, 523-526 (2002) 